\title{
TIẾP CẬN PHƯƠNG PHÁP HOÀN THIỆN VÀ HIỆN ĐẠI HÓA HỆ ĐỘ CAO QUỐC GIA
}

\author{
PGS. TSKH. HÀ MINH HÒA \\ Viện Khoa học Đo đạc và Bản đồ
}

\section{Tóm tắt:}

Bài báo khoa học này nghiên cứu phương pháp hoàn thiện và hiện đại hóa hệ độ cao quốc gia, theo đó trên cơ sở thực nghiệm mô hình quasigeoid VIGAC2014 cho thấy nó cho phép nhận được các độ cao chuẩn độ chính xác cao trong hệ độ cao HP72 trên cả đất liền và các hải đảo. Việc xác định các điểm độ cao hạng I, II quốc gia có đo GPS mà trên đó các hiệu giữa dị thường độ cao trọng lực (được xác định từ mô hình quasigeoid VIGAC2014) và dị thường độ cao GPS/thủy chuẩn có các giá trị đủ nhỏ sẽ cho phép giải quyết đồng thời các bài toán làm tương thích mô hình quasigeoid VIGAC2014 với mô hình quasigeoid GPS/thủy chuẩn và hiện đại hóa hệ độ cao HP72 dựa trên mô hình quasigeoid VIGAC 2014 được làm tương thích. Theo cách tiếp cận này, chúng ta sẽ hiệu chỉnh lại các giá trị độ cao chuẩn cho phù hợp với mô hình quasigeoid được xây dựng đối với các điểm độ cao hạng I, II mà trên đó các hiệu giữa dị thường độ cao trọng lực và dị thường độ cao GPS/thủy chuẩn có các giá trị lớn vượt hạn sai.

\section{1. Đặt vấn đề}

Việt Nam cũng như tất cả các quốc gia khác trên thế giới đã xây dựng và khai thác hệ độ cao quốc gia với mặt khởi tính là mặt biển trung bình nhiều năm trên một trạm nghiệm triều (được gọi là trạm nghiệm triều 0). Ví dụ, hệ độ cao Hải Phòng 1972 ở Việt Nam được xây dựng dựa trên mặt biển trung bình nhiều năm tại trạm nghiệm triều Hòn Dấu. Quá trình xây dựng hệ độ cao này bằng phương pháp đo thủy chuẩn truyền thống đòi hỏi kinh phí rất lớn của Nhà nước và thường kéo dài hang thập kỷ. Mạng lưới độ cao hạng I, II quốc gia ở Việt Nam đã được xây dựng, đo đạc bổ sung và hoàn chỉnh trong vòng 43 năm từ 1963 đến 2007 (xem Báo cáo tổng kết kỹ thuật (2008)). Tại Vương quốc Anh, theo tài liệu (Iliffe J.C., Ziebart M., Cross P.A., Forsberg R., Strykowski G., Tscherning C.C. (2003)), các mạng lưới độ cao quốc gia của nước Anh được xây dựng trong nhiều giai đoạn 1912 1921, 1936 - 1952 và 1952 - 1956. Hệ độ cao quốc gia của Canada là hệ CGVD 28 (the Canadian Geodetic Vertical Datum of 1928) với mặt khởi tính là mặt nước biển trung bình tại trạm nghiệm triều Rimouski, Quebec, Canada. Hệ độ cao này chính thức được sử dụng ở Canada vào năm 1935 với khoảng 70.000 mốc thủy chuẩn và chủ yếu nằm dọc các quốc lộ, các tuyến đường sắt ở Nam Canada (Veronneau M., J. Huang J. (2007)).

Trong suốt cả quá trình xây dựng và khai thác hệ độ cao quốc gia, tùy theo các khu vực địa lý khác nhau trên lãnh thổ quốc gia, nhiều mốc độ cao đã bị trồi, lún hoặc bị mất do các tác nhân tự nhiên (dịch chuyển đứng do chuyển động hiện đại của vỏ Trái đất, động đất, sự thay đổi của các mực nước ngầm v.v...) và các tác nhân nhân sinh (phát triển các hệ thống giao thông, phát triển các khu đô thị, sự lún bề mặt đất do khai thác mỏ, khai thác nước ngầm v...v). Sự thay đổi lớn độ cao của các mốc độ cao quốc gia dẫn tới sự khác nhau lớn giữa dị thường độ cao GPS/thủy chuẩn và dị thường độ cao trọng lực. Trong tài

Người phản biện: TS. Nguyễn Đình Thành 
liệu (Hà Minh Hòa (2014a)) đã thống kê sự thay đổi lớn độ cao của các mốc độ cao do các hiện tượng nêu trên ở Mỹ, Nhật Bản, Anh, Ai Cập, Thổ Nhĩ Kỳ, Trung Quốc. Ở Việt Nam, kết quả đánh giá độ chính xác của mô hình EGM2008 trên lãnh thổ quốc gia cho thấy trong tổng số 269 điểm độ cao hạng I được kiểm tra, chỉ có 185 điểm (chiếm 68,77\%) có các độ chênh giữa dị thường độ cao GPS/thủy chuẩn và dị thường độ cao trọng lực thỏa mãn hạn sai, trong khi đó có đến 17 điểm có độ chênh nêu trên nằm trong khoảng từ $0,40 \mathrm{~m}$ đến $0,82 \mathrm{~m}$ bao gồm các điểm I(BH-HN)33, I(BH-HN)39, I(BH-HN)42, I(BH-HN)48, I(HN-HP)2A, I(HN-HP)5, I(HN-HP)7, I(HN-HP)11A, I(DN-BMT)47A, I(HN-VL)4-1, I(HN-VL)6-1, I(HNVL)10A, I(LS-HN)22, I(LS-HN)29, I(LS-HN)36, I(HP-NB)14A, I(BH-TH)99.

Trong thời đại hiện nay, do thời gian và kinh phí khổng lồ, không một quốc gia nào trên thế giới hoàn thiện hệ độ cao quốc gia bằng cách đo lặp lại toàn bộ mạng lưới độ cao quốc gia bằng phương pháp đo thủy chuẩn truyền thống.

Vấn đề giải quyết bài toán hoàn thiện và hiện đại hóa hệ độ cao quốc gia dựa trên việc sử dụng mô hình quasigeoid quốc gia độ chính xác cao sẽ được nghiên cứu trong bài báo khoa học này.

\section{Giải quyết vấn đề}

\subsection{Xu hướng chung}

Như đã chỉ ra trong tài liệu (Hà Minh Hòa (2012a)), việc xác định mô hình quasigeoid độ chính xác cao (đối với các quốc gia hoặc nhóm nước sử dụng các hệ độ cao chuẩn, trong đó có Việt Nam) hoặc mô hình geoid độ chính xác cao (đối với các quốc gia hoặc nhóm nước sử dụng các hệ độ cao chính) là bài toán khoa học - kỹ thuật then chốt để xây dựng hệ độ cao và hệ quy chiếu tọa độ không gian hiện đại. Việc xây dựng mô hình quasigeoid độ chính xác cao trên lãnh thổ quốc gia không chỉ phục vụ việc hoàn thiện và hiện đại hóa hệ độ cao quốc gia, mà còn tạo cơ sở cho việc xây dựng hệ quy chiếu không gian quốc gia. Theo đánh giá trong tài liệu (Hà Minh Hòa (2013b)), khi xây dựng hệ quy chiếu không gian quốc gia, để đạt được độ chính xác tương đối của các tọa độ không gian ở mức $1.10^{-9}$, độ chính xác của mô hình quasigeoid quốc gia (hoặc mô hình geoid quốc gia) phải cao hơn $\pm 4 \mathrm{~cm}$. Hiện nay mô hình geoid CGG2013 (Canadian Gravimetric Geoid of 2013) của Canada đã đạt độ chính xác cao hơn $\pm 3 \mathrm{~cm}$ trên $80 \%$ phần lục địa, thêm vào đó mô hình geoid này được xây dựng dựa trên mặt geoid toàn cầu với thế trọng trường $\bar{W}_{0}=626368560 \mathrm{~m}^{2} \cdot \mathrm{s}^{-2}$ và các dữ liệu đo trọng lực chi tiết khu vực Bắc Mỹ, các mô hình trọng trường Quả đất GRACE, GOCE bao gồm EGM2008, EIGEN-6C, EIGEN-6C2, DIR3, TIM-R3, GOCOO3S và mô hình số độ cao DEM (Huang J., Véronneau M. (2013); Véronneau M. (2014)). Mô hình geoid trọng lực OSGM02 (Ordnance Survey Geoid Model of 2002) độ phân giải 1,5km x 1,5km của Vương quốc Anh được xây dựng dựa trên các dữ liệu đo trọng lực trên đất liền và trên biển của Cơ quan Khảo sát địa chất (BGS) của Anh, mô hình EGM96 của Mỹ, mô hình trọng lực KMS98 được xác định từ các dữ liệu altimetry của Đan Mạch, các dữ liệu trọng lực của Cục trọng lực quốc tế (BGI) của Pháp và mô hình số độ cao độ phân giải $50 \mathrm{~m}$ x 50m của Anh. Mô hình geoid OSGM02 đạt độ chính xác $\pm 3,2 \mathrm{~cm}$ trên cơ sở đánh giá trên 179 điểm cơ sở độ cao của nước Anh. 
Ngày nay, với việc sử dụng Khung quy chiếu quốc tế ITRF và lịch vệ tinh chính xác ở các mức $\pm 2,5 \mathrm{~cm}$ và $\pm 5,0 \mathrm{~cm}$ do Tổ chức IGS cung cấp, chúng ta có thể đạt được độ chính xác của độ cao trắc địa $m_{H}$ ở các mức $\pm 2,5 \mathrm{~cm}$ và $\pm 5,0 \mathrm{~cm}$. Với sai số trung phương $m_{\zeta}$ của dị thường độ cao $\xi$ ở mức $\pm 3 \mathrm{~cm}$ và sai số trung phương của độ cao trắc địa $m_{H}= \pm 2,5 \mathrm{~cm}$, từ công thức $m_{H^{\gamma}}= \pm \sqrt{m_{H}^{2}+m_{\zeta}^{2}} \quad$ chúng ta sẽ nhận được sai số trung phương của độ cao chuẩn được xác định từ công nghệ GNSS và mô hình quasigeoid ở mức $\pm 3,9 \mathrm{~cm}$. Độ chính xác này tương đương với độ chính xác của độ cao chuẩn hạng $\mathrm{I}$.

Chính từ cơ sở lý luận này đã hình thành 02 khuynh hướng rất cấp tiến trong việc xây dựng hệ độ cao hiện đại gắn liền với việc xây dựng mô hình quasigeoid quốc gia (hoặc mô hình geoid quốc gia). Chúng ta sẽ nghiên cứu các khuynh hướng này.

Khuynh hướng 1: Do rất nhiều mốc độ cao bị mất, sự thay đổi lớn độ cao của các mốc độ cao do các tác nhân tự nhiên và nhân sinh, thêm vào đó kinh phí và thời gian đo lặp mạng lưới độ cao rất lớn đối với lãnh thổ rộng lớn như Canada, nên nước này đã bỏ hoàn toàn hệ độ cao cũ CGVD 28 và xây dựng hệ độ cao mới CGVD2013 dựa trên mô hình geoid CGG2013 (Véronneau M., Huang J. (2007)). Trên đất nước Canada đã xây dựng 250 trạm thuộc hệ thống kiểm soát tích cực Canada (Canadian Active Control System), mạng lưới cơ sở Canada (Canadian Base Network) bằng công nghệ GNSS. Từ hệ thống này xác định các độ cao trắc địa độ chính xác cao trên tất cả các mốc độ cao quốc gia và tiếp theo xác định độ cao chính của các mốc độ cao quốc gia trong hệ độ cao mới CGVD2013 dựa trên mô hình geoid CGG2013 (Huang J., Véronneau M. (2013); Véronneau M. (2014)).

Khuynh hướng này đòi hỏi phải hiện chỉnh tất cả các đường bình độ trên các bản đồ địa hình các tỷ lệ của quốc gia.

Khuynh hướng 2: Xác định trên lãnh thổ quốc gia các mốc độ cao quốc gia ổn định nhất (các điểm này được gọi là các điểm cơ sở), tức các độ cao của các mốc này thực tế không bị thay đổi lớn dưới tác động của các nhân tố tự nhiên và nhân sinh, thêm vào đó trên các mốc độ cao này các hiệu giữa dị thường độ cao GPS/thủy chuẩn và dị thường độ cao trọng lực (hoặc các hiệu giữa độ cao geoid GPS/thủy chuẩn và độ cao geoid trọng lực) thỏa mãn hạn sai và có giá trị tương đối nhỏ. Sử dụng các hiệu nêu trên để làm tương thích (fitting) dị thường độ cao trọng lực với dị thường độ cao GPS/thủy chuẩn (hoặc độ cao geoid trọng lực với độ cao geoid GPS/thủy chuẩn) nhằm xác định mô hình quasigeoid (hoặc mô hình geoid) sát nhất với hệ độ cao hiện hành. Xu hướng này được áp dụng ở Anh với việc làm tương thích mô hình geoid trọng lực OSGM02 sát nhất với hệ độ cao hiện hành ODN (Ordnance Datum Newlyn) của nước Anh dựa trên 179 điểm độ cao ổn định theo phương pháp callocation trung phương (Forsberg R., Strykowski G., lliffe J.C., Ziebart M., Cross P.A., Tscherning C.C., Cruddace P. (2001); Iliffe J.C., Ziebart M., Cross P.A., Forsberg R., Strykowski G., Tscherning C.C. (2003)).

Theo khuynh hướng này, hệ độ cao hiện hành vẫn được giữ lại, tuy nhiên các độ cao của các điểm độ cao quốc gia (trừ các điểm cơ sở) phải được hiệu chỉnh lại nhờ mô hình quasigeoid quốc gia độ chính xác cao (hoặc mô hình geoid quốc gia độ chính xác cao) và công nghệ GNSS. 


\subsection{Cách tiếp cận của Việt Nam}

Khuynh hướng thứ hai được trình bày ở trên phù hợp hơn đối với lãnh thổ Việt Nam. Tuy nhiên ở Việt Nam gặp một khó khăn cơ bản: không đủ dữ liệu trọng lực trên lãnh thổ Việt Nam và vùng xung quanh để xác định vùng gần với bán kính $2^{\circ} 5-5^{\circ}$ trong bài toán xác định dị thường độ cao trọng lực theo tích phân Stokes. Hiện nay Viện Khoa học Đo đạc và Bản đồ mới hoàn thành việc xây dựng $C S D L$ trọng lực ở các vùng đồng bằng và trung du của Việt Nam bao gồm 67.790 điểm trọng lực chi tiết vào năm 2012, còn việc đo trọng lực chi tiết ở các khu vực vùng núi và trên biển sẽ được thực hiện trong giai đoạn 2015 - 2020. Việc thu thập đầy đủ các dữ liệu trọng lực ở các nước xung quanh Việt Nam là một điều rất khó khăn, đặc biệt ở Lào và Căm Pu Chia thực tế có thể coi chưa có các dữ liệu trọng lực.

Do đó, phương hướng thực tế hơn là sử dụng các dữ liệu mô hình trọng trường Quả đất EGM2008, độ cao chuẩn quốc gia và GNSS để xây dựng mô hình quasigeoid quốc gia khởi đầu. Tiền đề cho phương hướng này là xác định được thế trọng trường $\mathrm{W}_{0}=$ $62636847.2911 \mathrm{~m}^{2} . \mathrm{s}^{-2}$ với sai số trung phương $0.183 \mathrm{~m}^{2} \mathrm{~s}^{-2}$ của mặt geoid cục bộ sát nhất với mặt biển trung bình tại trạm nghiệm triều Hòn Dấu (xem các tài liệu Hà Minh Hòa và nnk (2012b); Hà Minh Hòa (2012c); Ha Minh Hoa (2013a); Hà Minh Hòa (2014c)). Từ đây chúng ta xác định được công thức xác định dị thường độ cao của mô hình Quasigeoid quốc gia khởi đầu (được đặt tên là VIGAC2014) theo công thức (Hà Minh Hòa (2014b); Hà Minh Hòa (2014c)):

$$
\hat{\bar{\zeta}}_{z}=\bar{\zeta}+\frac{\bar{W}_{0}-W_{0}}{10^{-5} \cdot \bar{\gamma}}+\delta \zeta_{n-z},
$$

ở đây $\bar{\xi}$ - giá trị từ mô hình trọng trường Quả đất EGM2008 tương ứng với mặt Geoid toàn cầu; - giá trị trung bình của gia tốc lực trọng trường chuẩn (đơn vị mGal) tại điểm cần xác định; $\delta \zeta_{n-z}$ - số cải chính chuyển dị thường độ cao từ hệ không phụ thuộc triều về hệ triều 0 và có dạng:

$$
\delta \zeta_{n-z}=\left(0,02871-0,08584 \cdot \sin ^{2} B\right)<m>,
$$

Còn $\mathrm{B}$ - vĩ độ trắc địa của điểm.

Đại lượng $\frac{\bar{w}_{0}-W_{0}}{10^{-5} \cdot \bar{\gamma}}=0.890 \mathrm{~m}$ là độ cao của mặt quasigeoid Hòn Dấu so với mặt quasigeoid toàn cầu và cũng bằng độ cao của mặt geoid Hòn Dấu so với mặt geoid toàn cầu, ở đây $\bar{W}_{0}=626368560 \mathrm{~m}^{2} \cdot \mathrm{s}^{-2}, \mathrm{~W}_{0}=62636847.2911 \mathrm{~m}^{2} \cdot \mathrm{s}^{-2}$. Đại lượng trên là đại lượng không đổi trên toàn bộ phần lục địa và lãnh hải của Việt Nam (xem chi tiết trong các tài liệu Hà Minh Hòa và nnk (2012b); Hà Minh Hòa (2012c); Ha Minh Hoa (2013a); Hà Minh Hòa (2014c)).

Việc sử dụng mô hình EGM2008 trong việc xây dựng mô hình quasigeoid quốc gia ở Việt Nam xuất phát từ thực tế là các nước trên thế giới đã nghiên cứu thử nghiệm mô hình này và thừa nhận rằng mô hình này cho các giá trị dị thường độ cao trọng lực phù hợp nhất với các giá trị dị thường độ cao GPS/thủy chuẩn (Thomas Gruber (2008)) và các kết quả nghiên cứu ứng dụng mô hình này thường xuyên được công bố trong tạp chí Newton's 
Bulletin của IAG (International Association of Geodesy) và IGFS (International Gravity Field Service). Ở Việt Nam mô hình EGM2008 cũng đang được nghiên cứu và sử dụng rộng rãi.

Khi sử dụng mô hình (1), mặt quasigeoid VIGAC2014 trùng với mặt geoid cục bộ tại trạm nghiệm triều Hòn Dấu, thêm vào đó mặt quasigeoid này không hoàn toàn song song với mặt quasigeoid toàn cầu tương ứng với mặt geoid toàn cầu do mặt quasigeoid VIGAC2014 đã được chuyển về hệ triều 0 , trong khi đó mặt quasigeoid toàn cầu tương ứng với hệ không phụ thuộc triều.

Để đánh giá độ chính xác của mô hình quasigeoid VIGAC2014 trên lãnh thổ Việt Nam, chúng ta phải lựa chọn trên lãnh thổ Việt Nam các điểm cơ sở là các điểm độ cao hạng $\mathrm{I}$ có đo GPS và các dữ liệu đo GPS được xử lý trong ITRF. Các tiêu chí lựa chọn các điểm cơ sở như sau (Hà Minh Hòa (2014b)):

- Các dị thường độ cao trọng lực $\hat{\hat{E}_{\text {re }}}$ được xác định theo công thức (1);

- Giá trị tuyệt đối của hiệu (độ lệch) $d=\left(\zeta_{z}-\hat{\bar{\zeta}}_{z}\right.$ giữa dị thường độ cao GNSS/thủy chuẩn $(\zeta)_{z}$ (đã được đưa về hệ triều) và dị thường độ cao trọng lực ${ }_{\frac{5}{2}}^{*}$ không được lớn hơn $1 \mathrm{~cm}$.

Cơ sở khoa học của tiêu chí 2 đã được chứng minh trong tài liệu (Hà Minh Hòa (2014b)). Trên cơ sở các tiêu chỉ nêu trên, trên phần đất liền của lãnh thổ Việt Nam đã chọn được 09 mốc độ cao hạng I làm các điểm cơ sở bao gồm các mốc (HN-VL)6-1, I(HNVL)28-1, I(HN-VL)64, I(HN-VL)72, I(VL-HT)98, I(VL-HT)158, I(BH-HN)33, I(BH-TH)65, I(BH-TH)122A. Nếu chúng ta gọi mặt quasigeoid quốc gia được xác định bởi các giá trị dị thường độ cao GPS/thủy chuẩn trên các điểm thuộc lãnh thổ Việt Nam là mặt quasigeoid GPS/thủy chuẩn, thì tại các điểm cơ sở mặt quasigeoid GPS/thủy chuẩn thực tế chênh nhỏ bỏ qua so với mặt quasigeoid trọng lực VIGAC2014.

Các kết quả đánh giá sai số trung phương của các độ chênh $d=(\zeta)_{z}-\hat{\bar{\zeta}}_{z}$ trên 09 điểm cơ sở được trình bày ở bảng 1 ở dưới đây.

Bảng 1

\begin{tabular}{|c|c|}
\hline Tên điểm & $\begin{array}{c}\text { Độ chênh } \\
d=(\zeta)_{z}-\hat{\bar{\zeta}}_{z} \\
\end{array}$ \\
\hline $\mathrm{m})$
\end{tabular}


Khi coi dị thường độ cao GPS/thủy chuẩn và dị thường độ cao trọng lực từ mô hình VIGAC2014 là các trị đo kép, độc lập, dựa trên bảng 1, chúng ta xác định được sai số trung phương của dị thường độ cao từ mô hình VIGAC2014 như sau:

$$
m_{\widetilde{\zeta}_{i}}= \pm \sqrt{\frac{\left[d_{i}^{2}\right]}{2 \cdot N}}= \pm \sqrt{\frac{0,000416}{18}}= \pm 0,005 \mathrm{~m} .
$$

Sai số trung phương được tính toán hoàn toàn nhỏ bỏ qua trong bài toán xác định mặt quasigeoid. Các điểm cơ sở này được coi là các điểm ổn định nhất về mặt địa chất kiến tạo trong toàn bộ các điểm độ cao hạng I quốc gia và được ký hiệu theo thứ tự $i=1,2, \ldots, n$. Trong trường hợp đang xem xét, $\mathrm{n}=9$.

Khi trên điểm cần xác định độ cao chuẩn trong hệ độ cao quốc gia có đo GPS và xử lý các dữ liệu đo GPS trong ITRF, chúng ta nhận được độ cao chuẩn $H_{z}$ trong hệ triều 0 theo công thức (xem trong tài liệu Hà Minh Hòa (2014c)):

$$
H_{z}=H_{n}+\left(0,602073-0,185592 \cdot \sin ^{2} \mathrm{~B}\right)
$$

ở đây $H_{n}$ - độ cao trắc địa của điểm trong hệ không phụ thuộc triều được xác định từ kết quả xử lý các dữ liệu GPS trong ITRF, B - vĩ độ trắc địa của điểm.

Khi đó, độ cao chuẩn $H_{z}^{\gamma}$ của điểm được xác định theo công thức:

$$
H_{z}^{\gamma}=H_{z}-\hat{\bar{\zeta}}_{z}
$$

ở đây dị thường độ cao trọng lực $\hat{\bar{\zeta}}_{z}$ được xác định từ mô hình quasigeoid VIGAC2014 theo công thức (1).

Bây giờ, để kiểm tra mô hình quasigeoid VIGAC2014 trên lãnh thổ Việt Nam, chúng ta xác định độ cao chuẩn của điểm $j$ bất kỳ từ $\mathrm{n}$ điểm cơ sở theo công thức:

$$
\left(\widetilde{H}_{j}\right)_{z}=\frac{\sum_{i=1}^{n}\left(H_{j}^{\gamma}\right)_{i}}{n},
$$

ở đây độ cao chuẩn

$$
\left(H_{j}^{\gamma}\right)_{i}=\left(H_{i}^{\gamma}\right)_{z}+\left\{\left(\bar{H}_{j}\right)_{z}-\left(\bar{H}_{i}\right)_{z}\right\}-\left\{\left(\hat{\bar{\zeta}}_{j}\right)_{z}-\left(\sigma_{i}\right)_{z}\right\}
$$

Việc so sánh độ cao chuẩn (2) với độ cao chuẩn (3) sẽ cho phép khẳng định cơ sở khoa học của việc xây dựng mô hình Quasigeoid quốc gia khởi đầu VIGAC2014 được nêu ở trên và đánh giá độ chính xác của mô hình này.

Trong tài liệu (Hà Minh Hòa (2014b)) đã tiến hành kiểm tra mô hình quasigeoid VIGAC2014 trên cơ sở sử dụng 04 điểm cơ sở I(HN-VL)64, I(HN-VL)72, I(VL-HT)98, I(VLHT)158 để chuyển độ cao chuẩn ra hai điểm GPS được bố trí tại hai trạm nghiệm triều trên các đảo Phú Quốc và Côn Đảo. Độ chênh của các độ cao chuẩn được xác định theo các công thức (2) và (3) chỉ ở mức $2 \mathrm{~mm}$.

Trong bài báo khoa học này, chúng ta sẽ tiếp tục kiểm tra mô hình quasigeoid VIGAC2014 khi xác định độ cao chuẩn trên khu vực vùng núi Việt Nam. Chúng ta sử dụng 


\section{Nghiên cứu}

04 điểm C004 (Điện Biên), C022 (Sơn La), C065 (Tuyên Quang) và C075 (Quảng Ninh) thuộc mạng lưới trắc địa địa động lực miền Bắc được đo liên tục trong 4 ngày đêm trong tháng 01/2013. Các dữ liệu GPS đo trên các điểm này được xử lý trong ITRF2008 tương ứng với ellipsoid WGS84 quốc tế bằng phần mềm Bernese v.5.2. và 3

Các khoảng cách từ 09 điểm cơ sở đến 04 điểm kiểm tra được trình bày ở các bảng 2

Bảng 2

\begin{tabular}{|c|c|c|c|c|c|}
\hline STT & Điểm i & Điểm j & $\begin{array}{c}\text { Khoảng cách Sij } \\
(\mathrm{m})\end{array}$ & Điểm j & $\begin{array}{l}\text { Khoảng cách Sij } \\
(\mathrm{m})\end{array}$ \\
\hline 1 & $\mathrm{I}(\mathrm{HN}-\mathrm{VL}) 6-1$ & \multirow{9}{*}{ C004 } & 307240,2 & \multirow{9}{*}{$\mathrm{C} 022$} & 169534,5 \\
\hline 2 & $\mathrm{I}(\mathrm{HN}-\mathrm{VL}) 28-1$ & & 355432,1 & & 206342,8 \\
\hline 3 & $\mathrm{I}(\mathrm{HN}-\mathrm{VL}) 64$ & & 501402,0 & & 357267,2 \\
\hline 4 & $\mathrm{I}(\mathrm{HN}-\mathrm{VL}) 72$ & & 537910,3 & & 392446,2 \\
\hline 5 & I(VL-HT)98 & & 1020279,2 & & 874099,4 \\
\hline 6 & I(VL-HT)158 & & 1228657,8 & & 1086642,9 \\
\hline 7 & $\mathrm{I}(\mathrm{BH}-\mathrm{HN}) 33$ & & 234445,9 & & 117693,9 \\
\hline 8 & $\mathrm{I}(\mathrm{BH}-\mathrm{TH}) 65$ & & 24977,2 & & 124646,3 \\
\hline 9 & $\mathrm{I}(\mathrm{BH}-\mathrm{TH}) 122 \mathrm{~A}$ & & 234207,4 & & 87669,2 \\
\hline
\end{tabular}

Bảng 3

\begin{tabular}{|c|c|c|c|c|c|}
\hline STT & Điểm i & Điểm j & $\begin{array}{c}\text { Khoảng cách Sij } \\
(\mathrm{m})\end{array}$ & Điểm j & $\begin{array}{c}\text { Khoảng cách Sij } \\
(\mathrm{m})\end{array}$ \\
\hline 1 & I(HN-VL)6-1 & \multirow{9}{*}{ C065 } & 129739,7 & \multirow{9}{*}{$\mathrm{C} 075$} & 98797,8 \\
\hline 2 & $\mathrm{I}(\mathrm{HN}-\mathrm{VL}) 28-1$ & & 227913,7 & & 172556,1 \\
\hline 3 & $\mathrm{I}(\mathrm{HN}-\mathrm{VL}) 64$ & & 400214,7 & & 315685,4 \\
\hline 4 & $\mathrm{I}(\mathrm{HN}-\mathrm{VL}) 72$ & & 426862,5 & & 329105,2 \\
\hline 5 & $\mathrm{I}(\mathrm{VL}-\mathrm{HT}) 98$ & & 888496,9 & & 749773,8 \\
\hline 6 & I(VL-HT)158 & & 1115155,2 & & 983925,3 \\
\hline 7 & $\mathrm{I}(\mathrm{BH}-\mathrm{HN}) 33$ & & 55593,0 & & 150797,1 \\
\hline 8 & $\mathrm{I}(\mathrm{BH}-\mathrm{TH}) 65$ & & 212343,0 & & 365502,5 \\
\hline 9 & $\mathrm{I}(\mathrm{BH}-\mathrm{TH}) 122 \mathrm{~A}$ & & 128658,9 & & 183736,5 \\
\hline
\end{tabular}

Các kết quả xử lý các dữ liệu đo GPS trong ITRF và xác định độ cao chuẩn theo công thức (2) được trình bày ở bảng 4 dưới đây. 
Bảng 4

\begin{tabular}{|c|c|c|c|c|}
\hline $\begin{array}{c}\text { Các điểm GPS thuộc } \\
\text { mạng lưới trắc địa địa } \\
\text { động lực miền Bắc đo } \\
\text { tháng } 01 / 2013\end{array}$ & \begin{tabular}{|c|} 
Các tọa độ trắc địa \\
trong ITRF2008 \\
tương ứng với ellip- \\
soid WGS84 quốc \\
tế \\
o , "
\end{tabular} & $\begin{array}{c}\text { Độ cao trắc địa của } \\
\text { các điểm tương ứng } \\
\text { với ellipsoid WGS84 } \\
\text { quốc tế trong hệ } \\
\text { triều } 0, \mathrm{~m}\end{array}$ & $\begin{array}{c}\text { Dị thường độ cao } \\
\text { của các điểm tương } \\
\text { ứng với mặt } \\
\text { Quasigeoid quốc gia } \\
\text { khởi đầu trong hệ } \\
\text { triều } 0, \mathrm{~m}\end{array}$ & $\mid \begin{array}{c}\text { Độ cao chuẩn của } \\
\text { các điểm so với mặt } \\
\text { Quasigeoid quốc gia } \\
\text { khởi đầu, m }\end{array}$ \\
\hline C004 & $\begin{array}{c}215532,784 \\
1031417,823\end{array}$ & 335,009 & $-31,764$ & 366,773 \\
\hline C022 & $\begin{array}{l}210143,806 \\
1041841,833\end{array}$ & 408,747 & $-28,949$ & 437,696 \\
\hline C065 & $\begin{array}{l}214837,766 \\
1052618,013\end{array}$ & 56,686 & $-28,755$ & 85,441 \\
\hline C075 & $\begin{array}{l}205915,178 \\
1064858,755\end{array}$ & $-10,756$ & $-24,324$ & 13,568 \\
\hline
\end{tabular}

Các kết quả tính chuyển độ cao chuẩn theo công thức (3) từ 09 điểm cơ sở được trình bày ở bảng 5 . (Xem bảng 5)

So sánh các bảng 4 và 5 chúng ta nhận thấy rằng khi xác định độ cao chuẩn theo mô hình quasigeoid VIGAC2014 theo công thức (2) và kiểm tra theo công thức (3), các giá trị độ cao chuẩn chỉ chênh nhau có $2 \mathrm{~mm}$.

Như vậy, các kết quả thử nghiệm một lần nữa cho phép chúng ta có thể làm 02 kết luận sau:

- Khẳng định lại một lần nữa kết luận: Trên lãnh thổ Việt Nam, độ cao giữa mặt quasigeoid cục bộ Hòn Dấu và mặt quasigeoid toàn cầu bằng $0,890 \mathrm{~m}$ và không đổi trên toàn bộ lãnh thổ;

- Mặt Quasigeoid quốc gia khởi đầu VIGAC2014 được xây dựng theo công thức (1) cho phép xác định độ cao chuẩn với độ chính xác cao trên toàn bộ lãnh thổ Việt Nam.

Một vấn đề nữa là sự tồn tại sai số bị bỏ qua (omission errors) trong dị thường độ cao trọng lực $\overline{L_{z}}$ được xác định từ mô hình trọng trường Quả đất EGM2008 trong công thức (1). Sai số bị bỏ qua nẩy sinh do lãnh thổ Việt Nam nằm trong khu vực "Fill - in", tức khu vực mà các dữ liệu trọng lực tại đó không được sử dụng để xây dựng mô hình EGM2008 và độ lớn của sai số bị bỏ qua có thể đạt tới mức $\pm(15-22) \mathrm{cm}$ (Pavlis N.K., Holmes S.A., Kenyon S.C. and Factor J.K. (2009)). Vậy chúng ta sẽ lý giải như thế nào khi các kết quả xác định độ cao chuẩn theo công thức (2) dựa trên mô hình quasigeoid VIGAC2014 và kiểm tra theo công thức (3) dựa trên 09 điểm cơ sở cho các giá trị độ cao chuẩn thực tế là trùng nhau?. Điều này chỉ có thể xẩy ra khi trên lãnh thổ Việt Nam, các giá trị dị thường độ cao trọng lực $\bar{\zeta}$ được xác định từ mô hình trọng trường Quả đất EGM2008 luôn chứa các sai số bị bỏ qua, nhưng giá trị của chúng là đại lượng không đổi và được ký hiệu là $\varepsilon$ Thật vậy, khi ký hiệu dị thường độ cao trọng lực

$$
\bar{\zeta}=\tilde{\zeta}+\varepsilon
$$


Bảng 5

\begin{tabular}{|c|c|c|c|c|c|}
\hline STT & Điểm i & Điểm j & $\left(H_{j}^{\gamma}\right)_{z}$ & Điểm j & $\left(H_{j}^{\gamma}\right)_{z}$ \\
\hline 1 & $\mathrm{I}(\mathrm{HN}-\mathrm{VL}) 6-1$ & \multirow{9}{*}{$\mathrm{CO04}$} & 366,779 & \multirow{9}{*}{$\mathrm{C} 022$} & 437,702 \\
\hline 2 & $\mathrm{I}(\mathrm{HN}-\mathrm{VL}) 28-1$ & & 366,764 & & 437,687 \\
\hline 3 & I(HN-VL)64 & & 366,779 & & 437,702 \\
\hline 4 & $\mathrm{I}(\mathrm{HN}-\mathrm{VL}) 72$ & & 366,772 & & 437,695 \\
\hline 5 & I(VL-HT)98 & & 366,764 & & 437,687 \\
\hline 6 & I(VL-HT)158 & & 366,773 & & 437,696 \\
\hline 7 & $\mathrm{I}(\mathrm{BH}-\mathrm{HN}) 33$ & & 366,764 & & 437,687 \\
\hline 8 & $\mathrm{I}(\mathrm{BH}-\mathrm{TH}) 65$ & & 366,781 & & 437,704 \\
\hline \multirow[t]{2}{*}{9} & $\mathrm{I}(\mathrm{BH}-\mathrm{TH}) 122 \mathrm{~A}$ & & 366,767 & & 437,690 \\
\hline & \multicolumn{2}{|c|}{ Trung bình } & 366,771 & & 437,694 \\
\hline STT & Điểm i & Điểm j & $\left(H_{j}^{\gamma}\right)_{z}$, & Điểm j & $\left(H_{j}^{\gamma}\right)_{z}$, \\
\hline 1 & $\mathrm{I}(\mathrm{HN}-\mathrm{VL}) 6-1$ & \multirow{9}{*}{ C065 } & 85,447 & \multirow{9}{*}{ C075 } & 13,574 \\
\hline 2 & $\mathrm{I}(\mathrm{HN}-\mathrm{VL}) 28-1$ & & 85,432 & & 13,559 \\
\hline 3 & I(HN-VL)64 & & 85,447 & & 13,574 \\
\hline 4 & $\mathrm{I}(\mathrm{HN}-\mathrm{VL}) 72$ & & 85,440 & & 13,567 \\
\hline 5 & $\mathrm{I}(\mathrm{VL}-\mathrm{HT}) 98$ & & 85,432 & & 13,559 \\
\hline 6 & I(VL-HT)158 & & 85,441 & & 13,568 \\
\hline 7 & $\mathrm{I}(\mathrm{BH}-\mathrm{HN}) 33$ & & 85,432 & & 13,559 \\
\hline 8 & $\mathrm{I}(\mathrm{BH}-\mathrm{TH}) 65$ & & 85,449 & & 13,576 \\
\hline 9 & $\mathrm{I}(\mathrm{BH}-\mathrm{TH}) 122 \mathrm{~A}$ & & 85,435 & & 13,562 \\
\hline & \multicolumn{2}{|c|}{ Trung bình } & 85,439 & & 13,566 \\
\hline
\end{tabular}

ở đây $\widetilde{\bar{\zeta}}$ - giá trị chính xác của dị thường độ cao trọng lực, thì từ công thức xác định thế trọng trường của mặt Geoid cục bộ tại trạm nghiệm triều 0 trên $\mathrm{M}$ điểm GPS/thủy chuẩn (xem các tài liệu Hà Minh Hòa và nnk (2012b); Hà Minh Hòa (2012c); Ha Minh Hoa (2013a); Hà Minh Hòa (2014e)):

$$
W_{0}=\frac{\sum_{i=1}^{M}\left(W_{0}\right)_{i}}{M},
$$

Khi lưu ý 


$$
\left(W_{0}\right)_{i}=\bar{W}_{0}+10^{-5} \cdot \bar{\gamma}_{i} \cdot\left(\bar{\zeta}_{i}-\zeta_{i}\right)=\bar{W}_{0}+10^{-5} \cdot \bar{\gamma}_{i} \cdot\left(\widetilde{\bar{\zeta}}_{i}-\zeta_{i}\right)+10^{-5} \cdot \bar{\gamma}_{i} \cdot \varepsilon
$$

Chúng ta có:

$$
W_{0}=\widetilde{W}_{0}+\frac{\varepsilon \cdot \sum_{i=1}^{M} 10^{-5} \cdot \bar{\gamma}_{i}}{M},
$$

ở đây $\bar{\zeta}_{i}$ và $\zeta_{i}$ là các giá trị dị thường độ cao trọng lực và GPS/thủy chuẩn trên điểm i, còn $\bar{W}_{0}=\bar{W}_{0}+\frac{\sum_{i=1}^{M} 10^{-5} \cdot \bar{\gamma}_{i} \cdot\left(\tilde{\bar{\zeta}}_{i}-\zeta_{i}\right)}{M}$ là giá trị thế trọng trường của mặt geoid cục bộ không bị ảnh hưởng của các sai số bị bỏ qua trong các giá trị dị thường độ cao trọng lực.

Tuy nhiên, như đã tính toán trong các tài liệu (Hà Minh Hòa và nnk (2012b); Hà Minh Hòa (2012c); Ha Minh Hoa (2013a); Hà Minh Hòa (2014c)), trên lãnh thổ Việt Nam các giá trị $10^{-5} \cdot \bar{\gamma}_{i} \quad$ thay đổi rất nhỏ và được coi như hằng số, điều này dẫn đến đại lượng $\frac{W_{0}-W_{0}}{10^{-5} \cdot \bar{\gamma}}=0.890 \mathrm{~m}$ là đại lượng không đổi trên toàn bộ lãnh thổ Việt Nam. Do đó, khi ký hiệu $10^{-5} \cdot \bar{\gamma}_{i}=10^{-5} \cdot \bar{\gamma}=$ const, từ công thức (5) chúng ta nhận được quan hệ $W_{0}=\widetilde{W}_{0}+\varepsilon \cdot 10^{-5} \cdot \bar{\gamma}$. Như vậy, trong đại lượng $\frac{\bar{W}_{0}-W_{0}}{10^{-5} \cdot \bar{\gamma}}=\frac{\bar{W}_{0}-\widetilde{W}_{0}}{10^{-5} \cdot \bar{\gamma}}-\varepsilon=0.890 \mathrm{~m}$ đã chứa sai số bị bỏ qua $\varepsilon$. Khi đó, lưu ý (4) chúng ta viết lại công thức (1) dưới dạng:

$$
\hat{\bar{\zeta}}_{z}=\widetilde{\zeta}+\varepsilon+\frac{\bar{W}_{0}-\widetilde{W}_{0}}{10^{-5} \cdot \bar{\gamma}}-\varepsilon+\delta \zeta_{n-z}=\widetilde{\zeta}+\frac{\bar{W}_{0}-\widetilde{W}_{0}}{10^{-5} \cdot \bar{\gamma}}+\delta \zeta_{n-z} .
$$

Chúng ta thấy rằng khi sai số bị bỏ qua $\varepsilon$ là đại lượng không đổi trên toàn bộ lãnh thổ quốc gia, nó sẽ bị loại bỏ trong công thức (1).

Một vấn đề tiếp theo là làm khớp mô hình quasigeoid VIGAC2014 với mô hình quasigeoid GPS/thủy chuẩn. Với mục đích xây dựng mô hình quasigeoid quốc gia với độ chính xác cao hơn $\pm 4 \mathrm{~cm}$, chúng ta sẽ phải xây dựng tiêu chí xác định độ lớn cho phép của các hiệu $d=(\zeta)_{z}-\hat{\bar{\zeta}}_{z}$ trên các điểm độ cao hạng $\mathrm{I}$, II quốc gia có đo GPS. Vấn đề này sẽ được trình bày trong bài báo khoa học tiếp theo.

\section{Kết luận}

Với việc kiểm tra, đánh giá mô hình quasigeoid khởi đầu VIGAC2014 trên lãnh thổ Việt Nam và tiếp tục làm chính xác nó nhờ làm khớp mô hình quasigeoid VIGAC2014 với mô hình quasigeoid GPS/thủy chuẩn, chúng ta có thể xây dựng hoàn thiện hệ độ cao HP72 bằng cách đo GPS và xử lý các dữ liệu đo GPS trong ITRF trên các điểm độ cao hạng $\mathrm{I}$, II quốc gia (trừ các điểm độ cao quốc gia đã được đo trong giai đoạn 2009 - 2010) và xác 
định các giá trị độ cao chuẩn của các điểm này dựa trên mô hình quasigeoid VIGAC. Chúng ta sẽ loại bỏ các giá trị độ cao chuẩn của các điểm độ cao quốc gia có độ chênh lớn so với độ cao chuẩn được xác định từ mô hình quasigeoid VIGAC. Sử dụng các hiệu $d=(\zeta)_{z}-\hat{\bar{\zeta}}_{z}$ có độ lớn đủ nhỏ để làm khớp mô hình quasigeoid VIGAC2014 với mô hình quasigeoid GPS/thủy chuẩn. Chúng ta sẽ đồng thời nhận được hệ độ cao HP72 được hiện đại hóa và mô hình quasigeoid quốc gia độ chính xác cao cuối cùng. $O$

\section{Tài liệu tham khảo}

[1]. Báo cáo tổng kết kỹ thuật (2008). Thiết kế kỹ thuật - dự toán bình sai tổng thể lưới độ cao nhà nước hạng I, II. Cục Đo đạc và Bản đồ Việt Nam. Hà Nội - 2008.

[2]. Forsberg R., Strykowski G., Iliffe J.C., Ziebart M., Cross P.A., Tscherning C.C., Cruddace P. (2001). OSGM02: A new geoid model of the British Isles. www.discovery.ucl.ac.uk>... >UCL.DISCOVERY

[3]. Hà Minh Hòa (2012a). Các phương hướng hoàn thiện hệ độ cao quốc gia. Tạp chí Khoa học Đo đạc và Bản đồ, No11, 03/2012, trg. 1 - 9.

[4]. Hà Minh Hòa và nnk (2012b). Nghiên cứu cơ sở khoa học của việc hoàn thiện hệ độ cao gắn liền với việc xây dựng hệ tọa độ động lực quốc gia. Đề tài khoa học và công nghệ cấp Bộ Tài nguyên và Môi trường giai đoạn 2010 - 2012. Hà Nội - 2012.

[5]. Hà Minh Hòa (2012c). Nghiên cứu xác định thế năng trọng trường thực $W_{0}$ của mặt Geoid cục bộ trùng với mặt biển trung bình tại trạm nghiệm triều Hòn Dấu. Báo cáo khoa học. Tuyển tập báo cáo Hội nghị Khoa học và Công nghệ "Trắc địa và Bản đồ vì sự nghiệp Tài nguyên và Môi trường". Viện Khoa học Đo đạc và Bản đồ - Hội Trắc địa, Bản đồ Viễn thám Việt Nam. Hà Nội - tháng 10/2012. Trg. 6-19.

[6]. Ha Minh Hoa (2013a). Estimating the geopotential value $W_{0}$ of the local geoid based on data from local and global normal heights of GPS/Leveling points in Vietnam. Geodesy and Cartography. Taylor \& Francis. UDK 528.21, doi:10.3846/20296991.2013.823705, V.39 (3): 99-105.

[7]. Hà Minh Hòa (2013b). Phương pháp bình sai các mạng lưới trắc địa quốc gia. Nhà Xuất bản Khoa học và Kỹ thuật, 244 trg., Hà Nội - 2013.

[8]. Hà Minh Hòa (2014a). Đánh giá độ chính xác của mô hình Quasigeoid EGM2008 trên lãnh thổ Việt Nam. Tạp chí Khoa học Đo đạc và Bản đồ, No20, 06/2014, trg. 1 -13.

[9]. Hà Minh Hòa (2014b). Phương pháp chuyển độ cao chuẩn nhà nước lên các mặt biển trung bình tại các trạm nghiệm triều trên các đảo xa. Báo cáo khoa học. Kỷ yếu Hội nghị Khoa học "Trắc địa và Bản đồ vì hội nhập quốc tế" ngày 08/07/2014. Viện Khoa học Đo đạc và Bản đồ, Hội Trắc địa, Bản đồ và Viễn thám Việt Nam trg. 2 - 15.

[10]. Hà Minh Hòa (2014c). Lý thuyết và thực tế của Trọng lực trắc địa. Nhà Xuất bản Khoa học và Kỹ thuật, 592 trg., Hà Nội - 2014.

[11]. Huang J., Véronneau M. (2013). Contribution of the GRACE and GOCE models to a geopotential - based geodetic vertical datum in Canada. Geophysical Research Abstracts, Vol. 15, EGU2013-10164. 
[12]. Iliffe J.C., Ziebart M., Cross P.A., Forsberg R., Strykowski G., Tscherning C.C. (2003). OSGM02: A New model for converting GPS-derived heights to local height datums in Great Britain and Ireland.

[13]. Pavlis N.K., Holmes S.A., Kenyon S.C. and Factor J.K. (2009). The Earth Gravitational Model 2008 (EGM2008). The OSU School of the Earth Sciences, Columbus, $\mathrm{OH}$, February, 12/2009, $51 \mathrm{p}$.

[14]. Véronneau M., Huang J. (2007). Moving to a Modernized Height Reference System in Canada: Rationale, Status and Plans. Natural Resources Canada. American Geophysical Union, Spring Meeting 2007. 1214 Geopotential theory and determination (0903), 1229 Reference systems. 007AGUSM.G33B.05V.

[15]. Véronneau M. (2014). The Canadian Geodetic Vertical Datum of 2013. Canadian Institute of Geomatics, Ottawa Branch, 29 April 2014, Natural Resources Canada, 33 p.

[16]. Thomas Gruber. (2008). Evaluation of the EGM2008 Gravity field by Mean of GPSLevelling and Sea surface topography solution. Institute of Astronomical and Physical Geodesy, Technical University of Munich, Germany. E-mail: Thomas.Gruber@bv.tumuenchen.de. $\mathrm{O}$

\section{Summary}

\section{Approach to method of perfection and modernization of state vertical system}

Assoc. Prof. Dr.Sc. Ha Minh Hoa

Vietnam Institute of Geodesy and Cartography

This scientific article researches method for perfection and modernization of state vertical system based on results of experiments of quasigeoid model VIGAC2014 that allows to obtain highly accurate normal heights on mainland and islands. Determination of GPS/first and second leveling points, on which differences between gravimetric height anomaly (got from the quasigeoid model VIGAC2014) and GPS/leveling height anomaly have enough small values, allows to solve parallel tasks of fitting of quasigeoid model VIGAC2014 to GPS/leveling quasigeoid model and modernization of the state vertical system HP72 based on fitted quasigeoid model VIGAC2014. By this approach, we will recorrect normal heights corresponding to constructed quasigeoid model for first and order leveling points on which differences between gravimetric height anomaly and GPS/leveling height anomaly have big values exceed error limit. $\mathrm{O}$

Ngày nhận bài: 25/8/2014. 